\title{
DANGERS D'IRRADIATION LORS D'UN VOL SPATIAL HABITE APPLICATION A UN PROJET TERRE-LUNE
}

\author{
Michel GENET * \\ (Manuscrit reģu le $\mathbf{2}$ janvier 1968)
}

\begin{abstract}
RÉSUMÉ
D'après les différents types de rayonnements rencontrés dans l'espace, nous avons essayé d'apprécier les doses que les cosmonautes seront amenés à recevoir lors d'un vol spatial habité.

Ces divers risques sont envisagés d'une façon générale et appliqués plus particulièrement au cas d'un projet terre-lune.

Il ressort de cette analyse que le principal danger vient des rayons cosmiques issus des éruptions solaires. En effet, les équivalents de dose déduite de ces flux de protons solaires pourraient être, même à l'intérieur de la cabine spatiale, de plusieurs centaines de rem. Ceci nous a conduit à discuter la détection et les moyens de protection possibles contre ce type de rayonnement.
\end{abstract}

\section{INTRODUCTION}

Dix ans après le lancement du premier satellite artificiel (octobre 1957), un programme très détaillé d'un voyage terre-lune est à l'étude. Tout permet de penser que la réalisation de ce projet aura lieu vers I969-1970. Parmi les nombreux problèmes que pose une telle entreprise, nous examinerons uniquement les dangers présentés par les différents rayonnements rencontrés dans l'espace.

Certains de ces rayonnements, comme les rayons cosmiques, sont étudiés depuis plus de so ans; par contre, les ceintures de Van Allen ne font l'objet de recherches précises que depuis mars 1958 , date de leur découverte par Explorer I et III [I].

A la suite de ces études, les positions des deux ceintures furent déterminées [2] [3] [4]; elles fixent ainsi l'apogée maximal, sur orbitre terrestre, des vols habités ne présentant pas de risques graves pour les cosmonautes.

Cet apogée ne doit pas dépasser 500 à $600 \mathrm{~km}$. Toutefois, il faut signaler que dans le programme de Gemini XI, en changeant d'orbite en cours de vol, quelques essais ont été réalisés jusqu'à I $368 \mathrm{~km}$ (septembre 1966).

Donc, même pour des orbites terrestres, on s'aperçoit que les vols habités peuvent comporter, entre autre risques, celui d'une irradiation. Envisageons l'importance de ce même risque dans le cas d'un aller-retour terre-lune. Paris Ve. 


\section{LE PROJET TERRE-LUNE}

Signalons, tout d'abord, que la majeure partie des renseignements dont on dispose correspond au projet américain Apollo. Nous utiliserons néanmoins certaines données générales fournies par l'U.R.S.S. sans que toutefois, ces renseignements puissent s'appliquer à un programme précis. Mais depuis dix ans, le chemin suivi indépendamment par les uns et les autres ne diffère, bien souvent, que dans les détails et il y a tout lieu de penser que le programme soviétique, sans le connaître, s'écarte probablement de très peu du programme américain.

Le projet Apollo prévoit donc que le voyage se déroulera de la façon suivante [s] :

La fusée Saturne V (3 0oo t environ) doit mettre trois hommes sur orbite terrestre. Cette étape durera quelques heures, voire quelques jours, pendant lesquels on procédera aux essais et aux vérifications des différentes manœuvres qui seront réalisées ensuite pour le voyage.

On peut supposer que l'apogée de l'orbite terrestre sera compris entre 300 et $600 \mathrm{~km}$ (parking orbit). De cette station, les trois hommes partiront à bord d'une unité de commande (Command Module : CM). C'est à partir de ce moment que débute véritablement le voyage qui durera 8,25 jours ( $196,6 \mathrm{~h}$ ) aller-retour. L'unité de commande se placera alors au départ de la terre, sur une orbite en direction de la lune, et arrivée au voisinage de celle-ci, l'unité de commande sera placée sur une orbite lunaire.

Avant-dernière phase du projet : un homme reste dans l'unité de commande, et les deux autres se rendent sur la lune à bord d'une unité d'excursion lunaire (Lunar Excursion Module: LEM). Il est prévu que leur temps de séjour à la surface de la lune ne dépassera pas 24 à $36 \mathrm{~h}$.

Dernière phase du voyage : les deux hommes regagnent l'unité de commande avec l'unité d'excursion lunaire en réalisant un rendez-vous sur orbite lunaire, et les trois cosmonautes à bord de l'unité de commande reviennent en direction de la terre où ils effectuent une rentrée, devenue maintenant classique, dans l'atmosphère.

Sans connaître, ni les latitudes de sortie ou de rentrée, ni les trajectoires exactes qui seront suivies, on peut quand même essayer d'évaluer les doses que recevront les cosmonautes en fonction des différents types de rayonnements qu'ils rencontreront au cours de ce voyage.

\section{LES DIFFÉRENTS RAYONNEMENTS RENCONTRÉS}

\section{I) GÉNÉRALITÉS SUR LES UNITÉS EMPLOYÉES}

D'une façon générale, nous donnerons soit les doses absorbées en rad soit les équivalents de dose en rem.

On sait que l'équivalent de dose (en rem) est numériquement égal au produit de la dose absorbée (en rad) par le facteur de qualité, le facteur de distribution de dose et d'autres facteurs adéquats. Pour l'irradiation externe avec des rayonnements pénétrants le facteur de qualité est seul à prendre en considération. 
Ce facteur de qualité, fixé à partir des valeurs expérimentales de l'efficacité biologique relative (EBR) est encore mal connu pour les divers rayonnements de l'espace et l'évaluation des équivalents de dose qui résultent de ces irradiations comporte une marge importante d'erreurs.

La détermination des EBR et du facteur de qualité de ces rayonnements constitue un problème important pour la radioprotection spatiale et conduit les expérimentateurs à étudier les effets biologiques des faisceaux de particules de haute énergie produits par les accélérateurs, particules dont la nature et l'énergie sont analogues à celles des rayonnements de l'espace[6-9].

\section{2) Les rayons cosmrques galactiques (RCG)}

Du point de vue irradiation, ce rayonnement représente dans l'espace interplanétaire un fond continu; en effet, il est distribué de façon isotrope et son intensité varie peu avec le temps. On considère que le débit de fluence est constitué à $85 \%$ de protons, 13 à $14 \%$ de particules $\alpha$ et $2 \%$ de particules de $Z \geqslant 3$.

L'abondance relative des différents éléments qui composent les RCG est voisine de celle des éléments dans l'univers, sauf pour quelques noyaux légers : $\mathrm{Li}, \mathrm{Be}, \mathrm{B}$, qui sont moins abondants. La densité de flux de particules entre la terre et la lune est comprise entre 2 et 4,5 particules. $\mathrm{cm}^{-2} . \mathrm{s}^{-1}$ [ro] dont les énergies se situent de 20 à $40 \mathrm{MeV} /$ nucléon jusqu'à ${ }^{12}{ }^{12} \mathrm{MeV} /$ nucléon. L'étude de ces rayons cosmiques est délicate étant donnée leur faible intensité; de ce fait, leur facteur de qualité est très difficile à déterminer Volynkin et al. [ $\mathrm{I}$ I] ont dressé un aperçu (tableau I) des doses calculées pour ce rayonnement, en prenant des valeurs des EBR tirées d'une étude de Rossi [ 12 ].

TABLEAU I

\begin{tabular}{|c|c|c|c|}
\hline $\begin{array}{l}\text { Particules } \\
\text { ou groupes } \\
\text { de noyaux }\end{array}$ & $\begin{array}{c}\text { Fluence } \\
\text { (part } . \mathrm{cm}^{-2} \\
. \mathrm{s}^{-1} \text { ) }\end{array}$ & $\begin{array}{l}\text { Dose } \\
\text { par jour } \\
\text { (m rad) }\end{array}$ & $\begin{array}{c}\text { Equivalent } \\
\text { de dose } \\
\text { par jour } \\
\text { (m rem) }\end{array}$ \\
\hline $\begin{array}{l}\text { Protons } \ldots \ldots \cdots \cdots \\
\text { Particules } \alpha \cdots \cdots \cdots \\
3 \leqslant Z \leqslant 5 \cdots \\
6 \leqslant Z \leqslant \text { ro } \ldots \cdots \cdots \\
Z>\text { ro } \ldots \ldots \cdots\end{array}$ & $\begin{array}{l}\mathrm{I}, 8 \\
0,4 \\
\text { I. } \mathrm{IO}^{-2} \\
2 . \mathrm{IO}^{-2} \\
\mathrm{I} \cdot \mathrm{rO}^{-2}\end{array}$ & $\begin{array}{l}4,9 \\
5,5 \\
0,4 \\
5,5 \\
5,2\end{array}$ & $\begin{array}{r}5,0 \\
5,5 \\
0,5 \\
33,0 \\
96,0\end{array}$ \\
\hline Total $\ldots \ldots \ldots \ldots$ & 2,24 & 21,5 & 140,0 \\
\hline
\end{tabular}

En tenant compte des rayonnements secondaires, Grigorev et al. [13] avancent les chiffres de 200 à 250 mrem par jour, ce qui représente environ ro fois l'équivalent de dose admissible pour un travailleur dans un laboratoire. Le vaisseau spatial comportera une protection de quelques $\mathrm{g} / \mathrm{cm}^{2}$ qui ne modifiera pratiquement pas l'intensité des RCG, et dans le cas de vols de longue durée, ce rayonnement présentera un danger non négligeable.

Pour des altitudes inférieures à $500 \mathrm{~km}$ environ, la situation est différente, car seules les particules d'énergie supérieure à is $\mathrm{GeV}$ atteignent la terre, les 
autres sont déviées par le champ magnétique terrestre, si bien qu'au cours des vols Gemini et Vostok, les doses reçues par les cosmonautes étaient peu importantes (tableau 2) [14] [Is].

TABLEAU 2

\begin{tabular}{|c|c|c|}
\hline $\begin{array}{c}\text { Cosmonautes } \\
\text { ou Vols }\end{array}$ & $\begin{array}{l}\text { Durée } \\
\text { du vol } \\
\text { (heures) }\end{array}$ & $\begin{array}{c}\text { Doses } \\
\text { moyennes } \\
\text { reçues pendant } \\
\text { le vol (m rad) }\end{array}$ \\
\hline 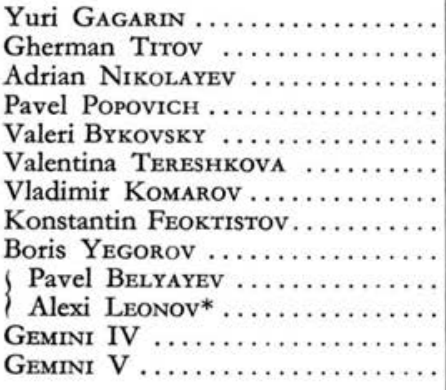 & $\begin{array}{l}1,8 \\
25,3 \\
95 \\
71 \\
119 \\
71 \\
24,3 \\
24,3 \\
24,3 \\
26 \\
26 \\
98 \\
191\end{array}$ & $\begin{array}{l}2,3 \\
10 \\
62 \\
46 \\
80 \\
44 \\
25 \\
29 \\
32 \\
75 \\
65 \\
50 \\
100\end{array}$ \\
\hline
\end{tabular}

* premier homme de l'espace.

On considère que ces doses sont dues en grande partie aux RCG. Ce rayonnement assure d'ailleurs à la surface de la terre un niveau d'irradiation constant de $0,4 \mathrm{rad}$ par an, soit I mrad par jour et donc une dose 20 fois plus petite que dans l'espace.

\section{3) Les ceintures de Van Allen (CVA)}

Elles sont au nombre de deux et intéressent le voisinage immédiat de la terre.

Les protons et les électrons qui les composent prennent naissance dans le bombardement cosmique de la haute atmosphère. Les compositions, les intensités et les contours de ces ceintures ont été déterminés à l'aide de compteurs GeigerMuller placés sur des orbites spéciales, dans les satellites suivants :

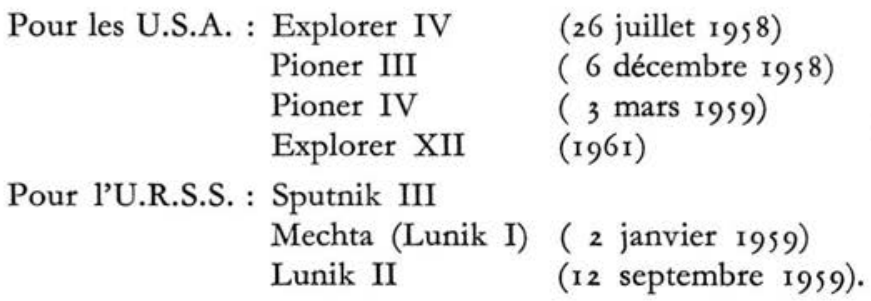




\section{a) La ceinture intérieure}

Elle se situe entre 600 et $10000 \mathrm{~km}$ et est constituée principalement de protons. Le flux de protons passe par un maximum à $3600 \mathrm{~km}$ et $7000-8000 \mathrm{~km}$; les énergies de ces particules sont comprises entre quelques $\mathrm{keV}$ et $100 \mathrm{MeV}$. Par exemple, pour des protons d'énergie $\left(E_{p}\right)$ supérieure à $40 \mathrm{MeV}$, le débit de fluence est de $2.10^{4} \mathrm{p} \cdot \mathrm{cm}^{-2} \cdot \mathrm{s}^{-1}$, mais pour $E_{p}>2 \mathrm{MeV}$, il passe à environ $10^{6} \mathrm{p} \cdot \mathrm{cm}^{-2} \cdot \mathrm{s}^{-1}$. L'énergie maximale des protons est de $700 \mathrm{MeV}$.

La densité de flux de protons ne varie pratiquement pas en fonction du temps. La figure I représente les différents débits de fluence des protons et des électrons en fonction de leur énergie et de l'altitude d'après Vernov et Chudakov. On a pu calculer que dans cette ceinture intérieure, le débit d'équivalent de dose à l'intérieur du vaisseau spatial $\left(\mathrm{r} \mathrm{g} / \mathrm{cm}^{2}\right)$ serait de $5 \mathrm{rem} / \mathrm{h}$.

Dans les mêmes conditions, les électrons de cette zone n'interviennent que par leur rayonnement de freinage; ils représentent une dose d'environ o, I rad/h.

\section{b) La ceinture extérieure}

A l'équateur, elle se trouve entre ro 000 et $50000 \mathrm{~km}$, mais pour des latitudes de $55^{\circ}$ à $70^{\circ}$, elle descend jusqu'à $300 \mathrm{~km}$.

Cette ceinture est avant tout constituée d'électrons provenant des injections des plasmas solaires. Contraitement à la ceinture interne, ses caractéristiques sont fortement influencées par les périodes d'activité solaire. On admet que ces électrons sont distribués de façon isotrope, leur énergie va de $20 \mathrm{keV}$ à plusieurs $\mathrm{GeV}$. Pour $\mathrm{E}_{e}>40 \mathrm{keV}$ on mesure par exemple, $\mathrm{Io}^{8}$ particules. $\mathrm{cm}^{-2} \cdot \mathrm{s}^{-1}$.

On estime que derrière une protection de $2 \mathrm{~g} / \mathrm{cm}^{2}$ la contribution de ces électrons serait de 20 rad par jour.

\section{c) Les ceintures artificielles.}

Pour vérifier les théories de l'injection des particules chargées dans le champ magnétique terrestre, plusieurs explosions nucléaires ont été provoquées à haute altitude. A la suite de ces expériences, on s'est aperçu que les dangers présentés par ces ceintures artificielles pouvaient être importants. Sur la proposition de Christofilos [16] au mois d'août et septembre 1958, trois petites bombes atomiques de $\mathrm{I}$ à 2 kilotonnes de TNT étaient lâchées à $500 \mathrm{~km}$ d'altitude. Ces expériences étaient suivies par le satellite Explorer IV qui indiqua qu'après les explosions, une ceinture de rayonnement (électrons) s'était placée entre les deux ceintures naturelles. Huit jours après l'explosion, cette ceinture artificielle n'était plus observable. Il est vraisemblable de penser qu'elle s'est trouvée éliminée vers les régions polaires de la terre.

L'emplacement de cette ceinture vérifie de façon satisfaisante les positions prévues théoriquement par VAN Allen. Ce mécanisme d'injection artificielle de particules chargées dans le champ magnétique terrestre fut appelé l'effet Argus.

Le 9 juillet $\mathrm{I}_{962}$, dans le cadre du projet Starfish, les américains font exploser en altitude une bombe A de puissance I,4 Mégatonnes de TNT. Cette fois, l'intensité et la largeur du flux d'électrons injectés sont bien supérieures à celles que l'on pouvait prévoir pour l'effet Argus. 
On suppose qu'au cours de cette expérience, le magnétisme terrestre a été perturbé, puisque des orages magnétiques furent enregistrés momentanément. A la suite de ces essais, trois satellites ont subi temporairement des avaries :. Traac, Ariel et Telstar I. On a pu calculer qu'au centre de la ceinture, la densité de flux d'électrons était d'environ $\mathrm{Io}^{14}$ particules. $\mathrm{cm}^{-2} \cdot \mathrm{s}^{-1}$. Leur énergie maximale était égale à $7 \mathrm{MeV}$. Aucun essai de cette sorte n'a eu lieu depuis.

La partie la plus stable de cette zone de rayonnement est située à une distance de 1200 à $4500 \mathrm{~km}$ au-dessus de l'équateur. Dans cette région de l'espace, on a mesuré les doses suivantes [17] :

TABLEAU 3

\begin{tabular}{c|c|c}
\hline \hline $\begin{array}{c}\text { Date de l'explosion: } \\
9 \text { juillet } 1962\end{array}$ & $\begin{array}{c}\text { Débit de dose en rad/h } \\
\text { avec un écran de : }\end{array}$ \\
\cline { 2 - 3 } & $0,4 \mathrm{~g} / \mathrm{cm}^{2}$ & $4,5 \mathrm{~g} / \mathrm{cm}^{2}$ \\
\hline 2 mois après l'explosion & 2000 & 3 \\
\hline 4 mois après l'explosion & $20000-30000$ & $30-35$ \\
\hline \hline
\end{tabular}

Il est logique de constater que le débit de dose augmente avec le temps, puisque la ceinture artificielle se trouve alimentée par les électrons provenant de la décroissance des produits de fission créés au moment de l'explosion.

A l'heure actuelle, le danger présenté par cette ceinture est équivalent à celui de la ceinture intérieure (protons), c'est-à-dire que le débit d'équivalent de dose est de 2 à $3 \mathrm{rem} / \mathrm{h}$ pour une protection de $2 \mathrm{~g} / \mathrm{cm}^{2}$. Mais ce débit diminue avec une période de $\mathrm{I}$ à 3 ans; on peut donc penser que d'ici 1970 les risques d'irradiation seront fortement réduits.

\section{4) Les Rayons Cosmiques Solaires (RCS)}

Ce rayonnement est surtout constitué de protons $(90 \%)$ et de particules $\alpha$ (10 \%), d'origine solaire. Ils proviennent des éruptions solaires, lesquelles sont associées aux taches solaires et donc aux périodes d'activité du soleil. Les éruptions ont lieu de façon presque aléatoire. Les radiations qui en résultent constituent dans l'espace un danger temporaire important, par suite de leur intensité et de leur énergie. On peut arbitrairement classer les éruptions solaires d'après l'énergie des particules émises en trois groupes : haute énergie, moyenne énergie et basse énergie. Les éruptions que nous citons correspondent surtout à la dernière période d'activité solaire, mais parmi les plus récentes qui ne sont pas classées, nous pouvons signaler celles du :

$$
\begin{aligned}
& 7 \text { juillet } 1966[18] \\
& 28 \text { août } \text { I }_{966} \\
& 2 \text { septembre } 1966 \\
& 28 \text { janvier } 1_{967} \\
& 28 \text { mai }{ }_{967}^{667}
\end{aligned}
$$


- Les éruptions de haute-énergie correspondent aux protons d'énergie supérieure à is $\mathrm{GeV}$, c'est-à-dire à ceux qui atteignent la surface de la terre. Ces éruptions durent quelques dizaines d'heures, leur intensité et leur fréquence sont faibles. On en compte environ une ou deux tous les cinq ans. Elles ont souvent lieu lors des périodes de croissance ou de décroissance de l'activité solaire.

Dans cette catégorie, on peut inclure les éruptions du :

$$
\begin{aligned}
& 28 \text { février } 1942 \\
& 7 \text { mars } 1942 \\
& 25 \text { juillet } 1946 \\
& 19 \text { novembre } 1949 \\
& 23 \text { février } 1956
\end{aligned}
$$

- Les éruptions de moyenne énergie (quelques $\mathrm{GeV}$ ) sont plus fréquentes, soit en moyenne 2 à 4 par an. Citons celles du Io et du 16 juillet 1959 et du 12 novembre 1960.

- Les éruptions de basse énergie (quelques centaines de $\mathrm{MeV}$ ) ont lieu assez fréquemment : ro à 12 fois par an. (22 août 1958, 10 mai 1959 et 14 juillet 1959).

Le calcul des doses relatives à ces éruptions nécessite la connaissance de nombreux paramètres, en particulier le facteur de qualité des protons solaires ainsi que leur distribution en énergie et en intensité pendant tout le temps de l'évènement.

Une éruption est caractérisée par le temps de montée et de descente du flux de particules. Elle peut se prolonger pendant plusieurs jours avec des intensités très variables. On ne peut donc pas donner une dose en fonction du temps, mais plutôt une dose totale intégrée pour un évènement donné. Le tableau 4 regroupe pour quelques éruptions les doses calculées pour des écrans d'épaisseur différente [19].

TABLEAU 4

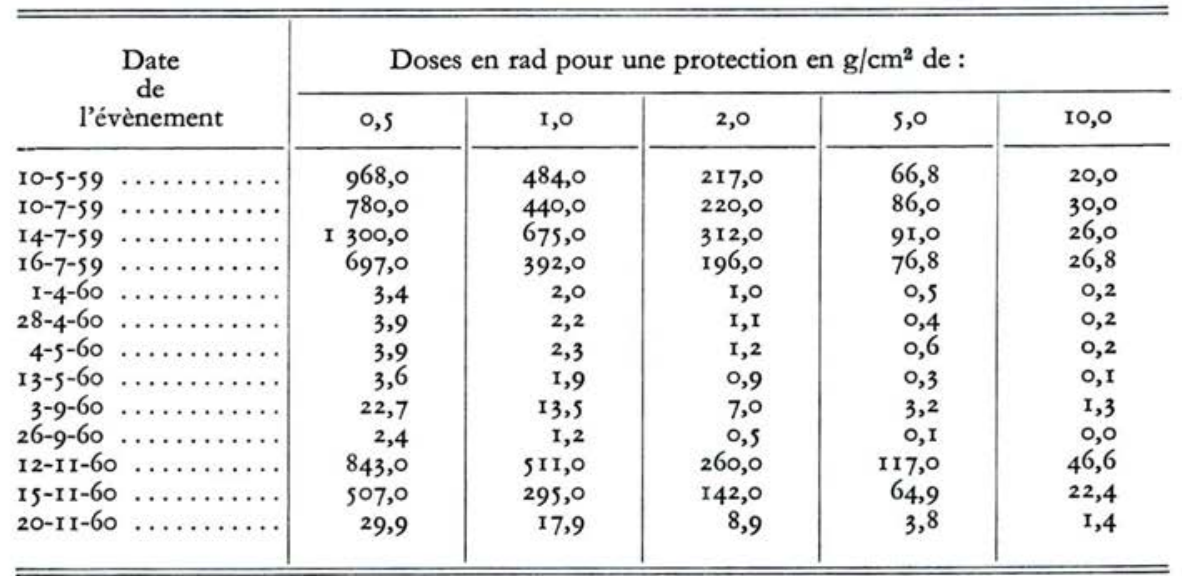


On s'aperçoit que la plupart du temps ces doses sont de plusieurs centaines de rad. On admet, de plus, que le facteur de qualité pour ces protons solaires, est compris entre I et 2 . Parfois, ces éruptions ont lieu à quelques jours d'intervalle et il n'est donc pas exclu que, pour un même vol, les cosmonautes rencontrent plusieurs vagues de protons provenant d'éruptions différentes.

\section{ESTIMATION DES ÉQUIVALENTS DE DOSE REÇUES POUR LE PROJET TERRE-LUNE}

Ces évaluations sont faites en supposant une protection de $2 \mathrm{~g} / \mathrm{cm}^{2}$ d'aluminium. Ces valeurs sont données par excès, car la protection du projet terre-lune sera supérieure à $2 \mathrm{~g} / \mathrm{cm}^{2}$.

\section{I) SUR ORBITE TERRESTRE}

Le champ magnétique terrestre protège les cosmonautes; ainsi, pour un séjour de quelques jours, en prenant un facteur de qualité égal à io pour certains RCG, l'équivalent de dose sera de l'ordre de quelques rem.

\section{2) LE VOYAGE TERRE-LUNE ( 8,25 JOURS)}

Tout en se libérant de l'attraction terrestre, les cosmonautes ne sont plus protégés par le champ magnétique terrestre. La dose due aux RCG se trouve doublée, de plus, les ceintures de Van Allen doivent être traversées et la probabilité d'une éruption solaire n'est pas exclue.

a) Les ceintures de Van Allen

On compte que les cosmonautes resteront environ is $\mathrm{mn}$ dans la ceinture intérieure et $\mathbf{2} \mathrm{h}$ dans la ceinture extérieure. Dans ces conditions, on peut calculer en choisissant des orbites convenables, que l'équivalent de dose total correspondant à la traversée normale des deux ceintures sera de 4 rem environ. Par contre, si un retour devait être effectué d'urgence sur une trajectoire très défavorable, la dose serait alors au maximum de $20 \mathrm{rem}[20]$.

\section{b) Les éruptions solaires}

Elles représentent de beaucoup le danger le plus grave. Pour une éruption de haute-énergie comme celle de février 1956 , nous avons vu que l'équivalent de dose aurait été de 400 rem environ. Suivant l'importance de l'évènement, cet équivalent de dose peut être compris entre quelques rem et 600 rem (i 4 juillet 1959).

\section{3) A la surface de la lune}

Si dans l'unité de commande les cosmonautes sont en sécurité relative, il n'en est pas de même dans l'unité d'excursion lunaire. Lorsqu'ils sortiront de cette unité, ils ne seront alors protégés que par la faible épaisseur de leur scaphandre individuel. Cette étape du voyage représente une phase critique où les cosmonautes se trouveront dans une situation très vulnérable. 
On estime que la dose due aux RCG est dix fois plus grande sur la lune que dans l'unité de commande. En cas d'éruption solaire, si les cosmonautes devaient rester à la surface de la lune, la dose serait, pour une éruption équivalente à celle du Io mai 1959, égale à 18099 rad [21]. Dans l'unité de commande, cette dose serait environ 100 fois plus faible. Le tableau s représente la contribution de chaque type de rayonnement pour les principales étapes du voyage terre-lune.

TABLEAU $s$

\begin{tabular}{|c|c|c|c|}
\hline \multicolumn{4}{|c|}{ Equivalents de dose en rem } \\
\hline \multirow{2}{*}{ EBR } & RCG & CVA & RCS \\
\hline & $5-10$ & $I-I, S$ & $I-2$ \\
\hline $\begin{array}{l}\text { Orbite terrestre } \\
\quad\left(2 \mathrm{~g} / \mathrm{cm}^{2}\right) \\
\text { Voyage terre-lune } \\
\quad\left(2 \mathrm{~g} / \mathrm{cm}^{2}\right) \\
\text { Surface } \mathrm{de} \mathrm{la} \mathrm{lune} \\
\quad\left(<\mathrm{I} \mathrm{g} / \mathrm{cm}^{2}\right)\end{array}$ & $\begin{array}{c}2 \text { à } 3 \\
2 \\
\text { (200 } \mathrm{m} \text { rem/jour })\end{array}$ & 4 & $\begin{array}{c}0-600 \\
0-36000\end{array}$ \\
\hline
\end{tabular}

L'examen de ce tableau fait ressortir l'importance du rayonnement cosmique solaire.

Le problème consiste donc à prévenir les cosmonautes de l'éruption solaire pour qu'ils aient le temps de regagner l'unité de commande, où la protection est mieux assurée.

Ceci nous amène à considérer les moyens de protection possibles contre des rayonnements.

\section{LES DIFFÉRENTS TYPES DE PROTECTION ENVISAGÉS}

\section{I) LES DIFFÉRENTES POSSIBILITÉS}

En premier lieu, l'épaisseur et la nature du matériau constituant l'unité de commande semble être d'une importance capitale. D'après les différents modèles de cabine proposés pour le projet Apollo, on pense maintenant que la protection sera environ de 3 à $7 \mathrm{~g} / \mathrm{cm}^{2}$ et que les matériaux retenus seront l'aluminium et le polyéthylène. Pour l'unité d'excursion lunaire, la protection serait inférieure à I $\mathrm{g} / \mathrm{cm}^{2}$.

Il semble aussi que l'agencement de l'unité de commande puisse jouer un rôle important; en effet, il est possible d'utiliser les substances emportées (carburant, eau) comme écran. Celà nécessite une répartition appropriée de ces éléments entre le flux de particules et la cabine habitée.

Les soviétiques ont même préconisé, étant donnée la brièveté des éruptions solaires, d'aménager à l'intérieur du vaisseau, un endroit de petite dimension, spécialement protégé par un blindage, où les cosmonautes seraient sans doute très mal à l'aise, mais en sécurité. 
De plus, on sait maintenant que suivant l'inclinaison de l'orbite du satellite, la dose croît rapidement avec la latitude. En choisissant judicieusement cette latitude en fonction de l'apogée de l'orbite, il est possible de réduire considérablement les doses reçues sur une orbite terrestre.

Signalons aussi que quelques projets de protection magnétique [22] [23] et électrostatique [24] [25] contre les particules chargées de haute énergie sont à l'étude; ceux-ci sont en général techniquement irréalisables pour le moment.

Enfin, précisons qu'actuellement des recherches sont poursuivies pour augmenter la radio-résistance de l'organisme à partir des composés chimiques et pharmaceutiques qui inhibent et transforment les produits radiolytiques en substances moins nocives [19].

\section{2) Protection spécifique contre chaque rayonnement}

\section{a) Les rayons cosmiques galactiques}

Etant donnée leur énergie, il n'y a aucune protection possible, et un écran de $3 \mathrm{~g} / \mathrm{cm}^{2}$ ne modifie pratiquement pas leur intensité. Donc, dès leur sortie du champ magnétique terrestre, les cosmonautes seront exposés à ces rayonnements de façon continue et inévitable.

\section{b) Les ceintures de Van Allen}

Cette fois, le problème est différent, la protection peut jouer un grand rôle. Les normes de la cabine Apollo prévoient que l'énergie des protons devra être au moins de ${ }_{3} 6 \mathrm{MeV}$ pour qu'ils parviennent à l'intérieur de la cabine. Dans ces conditions, les électrons de la ceinture extérieure n'interviendront que par l'intermédiaire de leur rayonnement de freinage.

\section{c) Les éruptions solaires}

Les risques étant importants, les moyens mis en œuvre pour dépister ces éruptions sont considérables [5] [26].

Rappelons que tout le problème consiste à gagner de vitesse le flux de protons solaires, de façon à pouvoir prévenir du danger les cosmonautes se trouvant sur la lune et à leur laisser le temps de réintégrer l'unité de commande.

Parmi les différents effets accompagnant les éruptions solaires trois d'entre eux ont été retenus pour permettre d'identifier celles-ci : les émissions radio du type IV, l'évolution visible de l'éruption, les répercussions au voisinage de la terre.

Les émissions radio du type IV, dans le domaine de quelques milliers de mégacycles, constituent les premiers renseignements en provenance de l'éruption. Elles seront détectées quelques minutes après l'éruption et on sait qu'il y a 90 à $100 \%$ de chances pour qu'elles soient associées à une éruption. Mais seulement une éruption sur trois est accompagnée d'un flux de protons qui atteindra quelques heures après le système terre-lune. Pendant le projet Apollo, trois radiotélescopes suivront en permanence ces émissions sur les trois fréquences suivantes: 1400 , 2900 et $4900 \mathrm{MHz}$. Immédiatement après la détection de l'éruption, celle-ci sera observée par 7 télescopes optiques qui examineront le développement de 
l'explosion solaire et essaieront de prévoir son évolution. Quelques heures après l'éruption, trois stations situées en Amérique du Nord se chargeront de déterminer le débit de fluence énergétique des protons en provenance du soleil. Ces stations s'intéressent aux modifications apportées par le flux de protons dans la couche $\mathrm{D}($ s० à $90 \mathrm{~km})$ de l'ionosphère.

Cet immense réseau de détection est en place depuis 1966 . Il représente au total I $_{3}$ stations au sol, s bateaux et 8 avions répartis sur l'ensemble du globe terrestre. Actuellement, la centralisation de tous ces renseignements et leur transmission au centre de contrôle du Projet Apollo est en cours. Cet ensemble constitue le SPAN (Solar Particle Alert Network). Il est amplement décrit dans un rapport de la NASA [s], il sera entièrement au point en juin 1968. On peut ajouter que ce réseau terrestre sera sans doute doublé d'un réseau de satellites chargés de confirmer les observations obtenues à la surface de la terre.

Un des premiers éléments de ce réseau est Explorer 34, lancé le 24 mai 1967; son apogée est de $325000 \mathrm{~km}$ et son périgée de $300 \mathrm{~km}$. Il s'appelle " sonnette d'alarme "). Il est chargé de détecter les éruptions solaires en étudiant les interactions des " vents solaires " et de la magnétosphère.

De plus, on sait maintenant par Pioneer $\mathrm{V}$ que le flux de protons solaires est suivi d'une brusque augmentation du champ magnétique interplanétaire (le $\mathrm{I}^{\text {er }}$ avril 1960 , celui-ci passait de $2,7 \gamma$ à plus de $50 \gamma)\left(\mathrm{I} \gamma=10^{-5}\right.$ gauss). Il est donc fort probable que d'autres lancements auront lieu dans le but de suivre ces variations de champ magnétique.

Indépendamment des mesures physiques, le calcul de probabilité permet d'estimer, pour un vol d'une semaine, les chances de rencontrer une éruption d'un type donné :

I6 \% pour une éruption de basse énergie

$5,8 \%$ pour une moyenne énergie et

$0,3 \%$ pour une haute énergie.

De plus, les soviétiques prétendent que, d'après le calcul, ils sont en mesure de prédire, avec $75 \%$ de réussite, une éruption deux à trois jours avant qu'elle n'ait lieu.

On s'aperçoit que le projet terre-lune est conditionné par les périodes d'activité solaire. On peut, en fonction de ce paramètre, essayer de prévoir les dates optimales de lancement de la cabine Apollo.

\section{LES PRÉVISIONS}

Le soleil connaît environ tous les onze ans une activité importante; celà se traduit surtout par l'augmentation du nombre de taches que l'on peut dénombrer à sa surface, mais aussi par la fréquence plus grande des éruptions solaires.

La figure 2 [27] représente l'allure théorique du cycle du soleil de 1964 à 1978. L'examen de cette courbe montre que nous sommes dans une très mauvaise période, puisque de 1967 à 1970 , nous nous trouvons dans une phase croissante de l'activité solaire, c'est-à-dire à un moment où une éruption de haute énergie peut se produire. 
Techniquement, il semble qu'à partir de i969-70 le projet Apollo soit réalisable. Il est impensable que la NASA attende 1976 pour trouver une activité solaire minimale. Le lancement aura donc lieu dans des conditions très défavorables; il est indispensable que le réseau de détection des éruptions soit le plus efficace possible.

En ce qui concerne le mois du lancement, il est possible d'avancer quelques propositions. En effet, R. PAY [28] a reporté sur un graphique (fig. 3) les dates et les années des principales éruptions depuis 1942 et il se trouve qu'entre le is mai et le 30 juin, ainsi qu'entre le er $^{\text {er }}$ décembre et le is janvier de chaque année, aucune éruption n'a eu lieu. Ceci n'est sans doute dû qu'à une pure coïncidence, mais il n'est pas impossible que cette constatation joue sur la date de lancement de Saturne V.

\section{CONCLUSION}

L'analyse des différents dangers d'irradiation pour un voyage spatial habité montre que pour une mission de deux semaines, les risques inévitables et permanents dus aux rayons cosmiques galactiques sont sans gravité. Il en est de même lors de la traversée des ceintures de Van Allen, puisque la protection du vaisseau spatial assure une dose inférieure à 20 rem, même dans le cas d'un retour forcé. Le véritable danger provient des protons solaires pour lesquels les doses, même à l'intérieur de la cabine, pourraient atteindre plusieurs centaines de rem. Dans le cadre du projet Apollo, un important dispositif terrestre et spatial a donc été mis en place en vue de renseigner les cosmonautes sur les dangers encourus.

A l'heure actuelle, ces dangers représentent une cause d'échec non négligeable et de ce point de vue, la norme de sécurité exigée est de $99,5 \%$.

Précisons aussi que les estimations que l'on peut faire sont sans doute entachées d'erreur très grande. En effet, on ne connaît pas le facteur de qualité de certains rayonnements comme la composante en noyaux lourds des RCG [29]. De plus, lorsque ces facteurs sont connus, ils sont déterminés sur des animaux et le plus souvent dans des conditions terrestres qui risquent donc de fausser les évaluations. On peut penser que les facteurs d'ambiance des cosmonautes: les réactions de tension émotionnelle, l'état d'apesanteur, les accélérations et décélérations diverses, etc. peuvent diminuer la résistance de l'homme au rayonnement.

De nombreuses recherches sont donc nécessaires avant que l'on puisse extrapoler les résultats que l'on possède à la surface de la terre sur des animaux, aux conditions des cosmonautes dans une capsule spatiale.

Enfin, si les problèmes semblent presque résolus dans le cas d'un voyage terre-lune de is jours, on conçoit difficilement un séjour prolongé à la surface de la lune (125 à $270 \mathrm{~m}$ rem par jour) ainsi qu'un voyage interplanétaire

Mars, Vénus, Mercure, sont à 400 jours de la terre; dans ces conditions, les risques s'évaluent à plusieurs centaines de rem [30] [3r] et, à l'heure actuelle, les problèmes radiobiologiques posés par de tels voyages ne semblent pas résolus.

\section{BIBLIOGRAPHIE}

[r] Van Allen J.A. Papier présenté au cour d'une réunion du « National Academy of Science and American Physical Society ". (1er mai 1958).

[2] Van Allen J.A. et Frank L.A. Nature, 1959, 183, 430. 
[3] Van Allen J.A. et Frank L.A. Nature, 1959, I84, 2 I9.

[4] Vernov S.N., Chudakov A.Y., Vakulov P.V. et Logachev Y.I. Doklady - Akad. Nauk. SSSR. 1959, 125, 304.

[s] Proceedings of the Apollo unified S. Band Technical Conference. (1965, I4-Is juillet). NASA - SP - 87. 135 .

[6] Bonet-Maury P., Kann T., Wembersies A., Choquet R., Bernet A., Sagey J. (de) et CIEUR L. I st Symposium on the biological interpretation of dose from accelerator produced radiation. Berkeley California, 13-16 March 1967.

[7] Legeay G., Tardy-Joubert Ph. et Botrom N. (de). Health Physics, r 966, i 2, 8, 1177.

[8] Bonet-Maury P., Deysine A., Frilley M. et Stefan C. C.R. Acad. Sci., Paris, r96o, 251, 3087.

[9] Bonet-Maury P., Baarli J., Kahn T., Dardenne G., Frilley M. et Deysine A. Biological effetcs of neutron and protons irradiations. IAE $A$, Vienne, 1964, 26r.

[io] Vernov S.N., Chudakov N.E., Vakulov P.V. et Logachev Y.I. Doklady. Akad. Nauk. SSSR, 1959, 125, 2.

[ir] Volynkin Y.M., Antipov A.V., Guda V.A., Nikrtin M.D. et Saksonov P.P. Papier présenté au XVe Congrès International d'Astronautique, Varsovie 1964 et NASA - TTF 279.

[1 2] Rossi H. Proceedings of a symposium at Harwell, 1962, 10-14 décembre. Neutron Dosimetry, I 963 , Vol. II.

[13] Grigorev Y.G., Kovalev E.E., Lebedinskiy A.V., Nefedov Y.G., Vysotskiy V.G., Ryjov N.I., Markelov B.A., Smirennyy L.N., Dudkin V.E., Derbeneva N.N. 2e Symposium International sur les problèmes fondamentaux concernant les facteurs d'ambiance rencontrés par l'homme dans l'espace. Paris, 1965, I4-18 juin, Preprint no 19.

[14] Bazykin V. Aerospace Medicine, 1965, 36, 12, I 194 .

[is] Schaeffer H.J. et Sullivan J.J. Aerospace Medicine, I967, 38, I, I.

[16] Christofilos N.C. J. Geophys. Res., I959, 64, 869.

[17] Clark B.C. et Adams D.A. Fifth International Space Science Symposium. Florence, mai 1964, 97.

[ir] Heristchi D.J., Kangas J., Kremser G., Legrand J.P., Masse P., Palous M., Pfotzer G., Riedler W. et Wilhelm K. SFARMO, Bulletin no 4, décembre 1966.

[19] Aerospace Medicine, 1965, 36, 2, section II.

[20] Aвel J. Preprint Amer. Astron. Soc., 1962, 37, 25.

[2I] Russa K S.L. J. Spacecraft, I964, I, 3 .

[22] Bernert R.E. et Stekly Z.J. 2nd Symposium on Protection against radiations in space. Gatlinburg, Tennessee, 1964, I 2-I4 October, NASA, SP-71, 199.

[23] Levine S.H. et Lepper R. American Institute of Aeronautics and Astronautics. $A I A A$ paper, no 66-513, 1966, june 27-29.

[24] Vogler F.H. 3ist Annual Meeting New York, Institute of the Aerospace Sciences. IAS paper, $\mathrm{n}^{\circ} 63-\mathrm{I} 2,1963$, january $2 \mathrm{I}-23$.

[25] Felten J.E. J. Astron. Sc., I964, I I, I, I6.

[26] LAFOND C.D. Missiles and Rockets, 1964, Is, 3, 18.

[27] J.W. Haffner. American Institute of Aeronautics and Astronautics. AIAA paper, no 65497, 1965, july 26-29.

[28] PAY R. Missiles and Rockets, 1964, 15, 3, 22.

[29] Schaeffer H.J. Aerospace Medicine, 1966, 37, I, I et Health Pbysics, 1967, 13, 327.

[30] Kotrler C.F. J. Astron. Sc., 1966, 13, 4, 133.

[3I] Savin R.C., Deerwester J.M. et MASCy A.C. J. Spacecraft, 1967, 4, 2, 249. Pour une bibliographie plus complète, consulter notre référence [19], (286 références). 


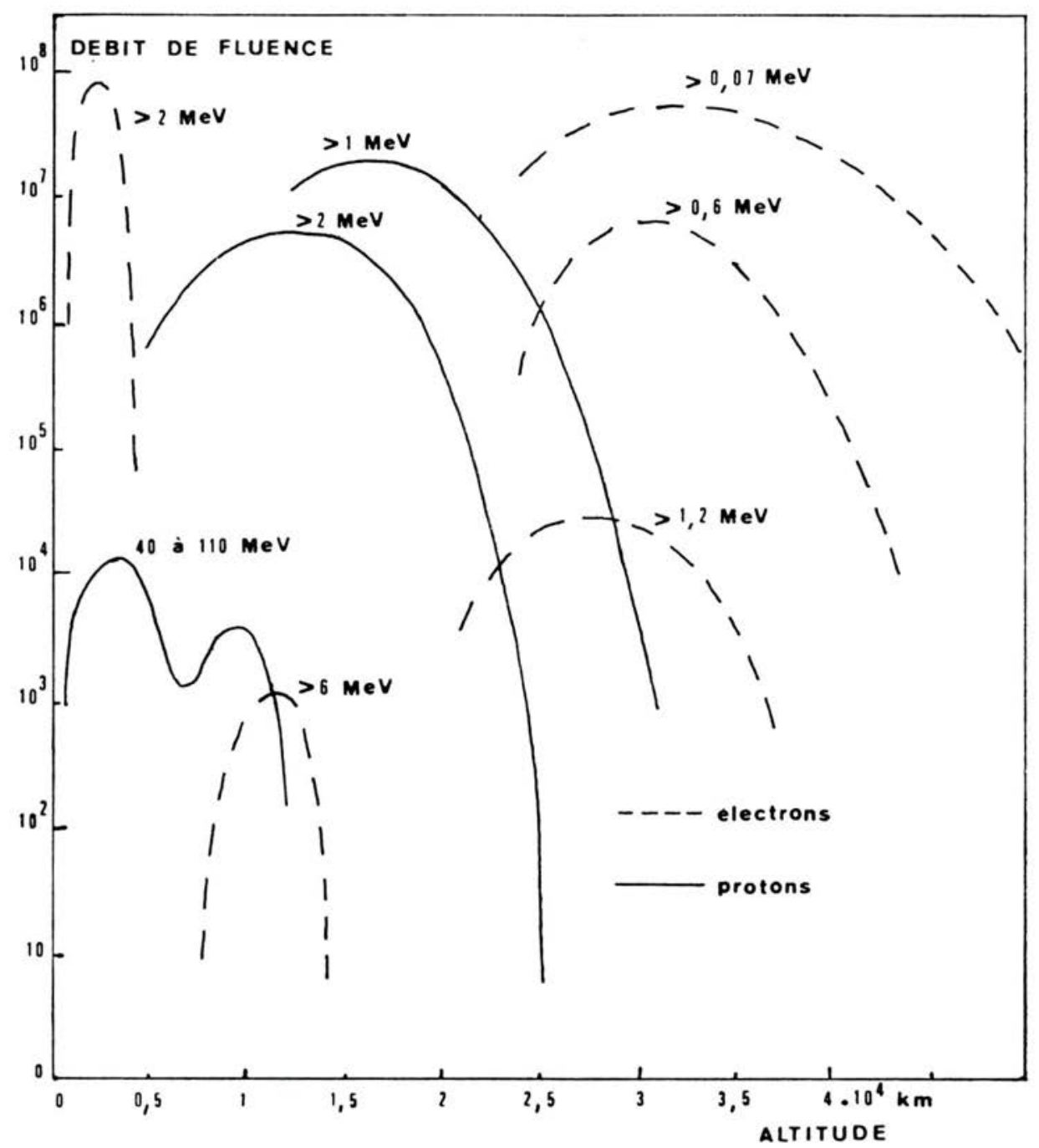

FIG. I. - Débits de fluence des électrons et des protons de différentes énergies dans les ceintures de Van Allen, en fonction de l'altitude. 


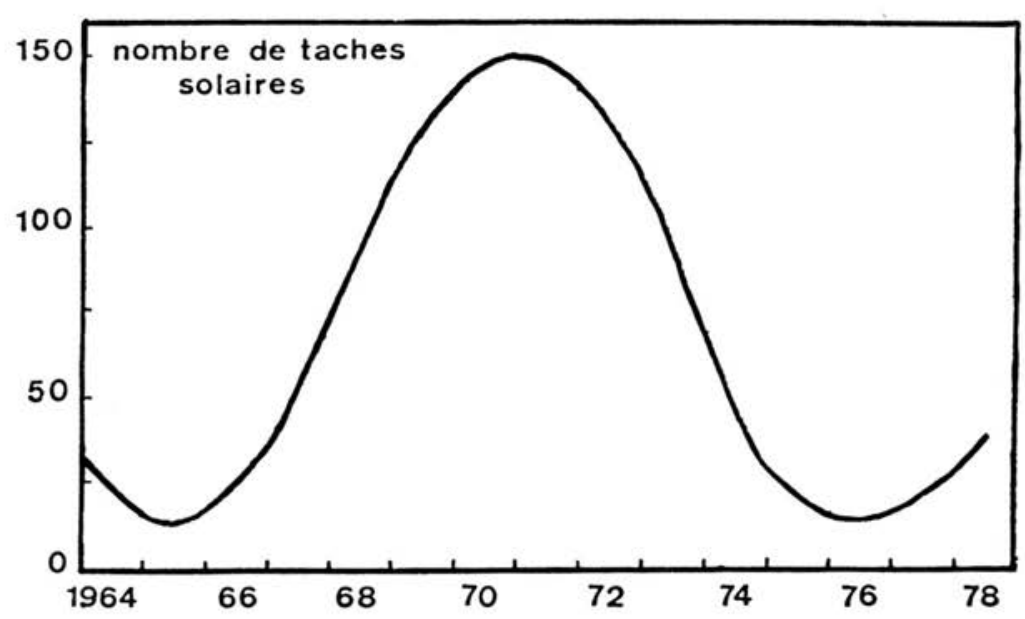

FIG. 2. - Variation prévue de l'activité solaire de 1964 à 1978.

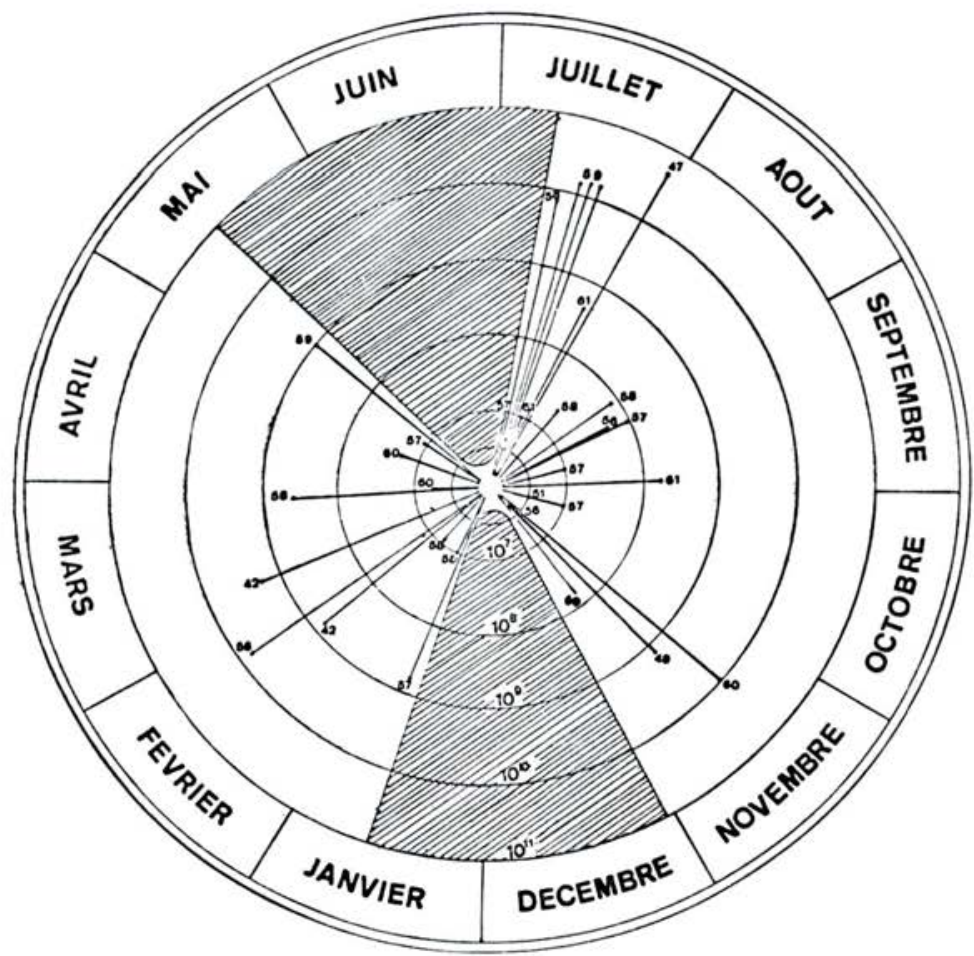

Fig. 3. - Fluence des particules en fonction de la date des éruptions solaires. 